\title{
A zinc finger protein Zfp521 directs neural differentiation and beyond
}

\author{
Sanbing Shen*, Jin Pu, Bing Lang and Colin D McCaig
}

\begin{abstract}
Neural induction is largely considered a default process, whereas little is known about intrinsic factors that drive neural differentiation. Kamiya and colleagues now demonstrate that a transcription factor, Zfp521, is capable of directing embryonic stem (ES) cells into neural progenitors. They discovered that Zfp521 transcripts were enriched in early neural lineage of ES cell differentiation. Forced expression of Zfp521 turned ES cells into neural progenitors in culture conditions that would normally inhibit neural differentiation. Zfp521 was expressed in mouse embryos during gastrulation. The protein was shown to associate with a co-activator p300 and directly induce expression of early neural genes. Knockdown of the Zfp521 by shRNA halted cells at the epiblast stage and suppressed neural differentiation. Zfp521 is a nuclear protein with 30 Krüppel-like zinc fingers mediating multiple protein-protein interactions, and regulates transcription in diverse tissues and organs. The protein promotes proliferation, delays differentiation and reduces apoptosis. The findings by Kamiya and colleagues that Zfp521 directs and sustains early neural differentiation now opens up a series of studies to investigate roles of Zfp521 in stem cells and brain development of mice and men.
\end{abstract}

Human embryonic stem (ES) cells offer tremendous opportunities for regenerative medicine, because they are capable of indefinite self-propagation and of differentiating into any cell types of three germ layers [1]. Significant progress has been made in understanding the maintenance of ES cells, and now induced pluripotent stem cells can be generated from most (if not all) somatic cells with the defined transcription factors c-MYC, KLF4, SOX2 and OCT4 [2] and/or small molecules [3]. A better

*Correspondence: Sanbing.Shen@abdn.ac.uk

School of Medical Sciences, Institute of Medical Sciences, University of Aberdeen, Foresterhill, Aberdeen AB25 2ZD, UK understanding of how to derive specific cell types from stem cells will increase the rate of translation of basic laboratory work to the clinic for patient-tailored therapy.

\section{Neural induction in embryos and ES cells}

Formation of the central nervous system has drawn the attention of developmental biologists for almost a century since Spemann and Mangold discovered that the dorsal lip of the blastopore was capable of inducing neural differentiation in Xenopus gastrula [4]. A list of Spemann organizers has been identified that to date includes Noggin, Gremlin, Cerberus, Twisted Gastrulation and Chordin. They are secreted molecules that directly bind and antagonize bone morphogenetic proteins (BMPs) $[5,6]$.

BMPs bind/activate specific high-affinity type I and type II Ser/Thr kinase receptors, including ALK1 to ALK7, ActRII, ActRIIB, T $\beta$ RII, BMPRI and BMPRII, and signal through the canonical SMAD pathways. BMP signaling pathways are present in ES cells and readily inhibit neural differentiation. When BMP inhibition is removed, the majority of surviving cells differentiate into neurons. Neural induction is therefore considered a default process that takes place autonomously and intrinsically [5]. Little is known, however, about the intracellular factors involved.

Kamiya and colleagues now report in Nature, with compelling evidence, that a zinc finger (ZF) nuclear protein, Zfp521, is a key intrinsic molecule capable of directing ES cells to neural progenitors [7]. They first knocked-in a GFP reporter gene into a neural precursor locus Sox 1 and cultured embryoid bodies for 3 days of default neural differentiation. They separated green (neural) and nongreen cells by flow cytometry, and identified 104 genes that were expressed highly in GFPpositive cells but were low in non-GFP cells. One of these genes, Zfp521, was able to turn ES cells into Sox1-GFPpositive neural progenitors by overexpression, either in the presence of BMPs or $10 \%$ FCS, which would normally inhibit neural differentiation.

Next, Zfp521 was shown to directly activate early neural genes such as Sox1, Sox3 and N-cadherin by working together with the co-activator p300. Knockdown of 


\section{(a)

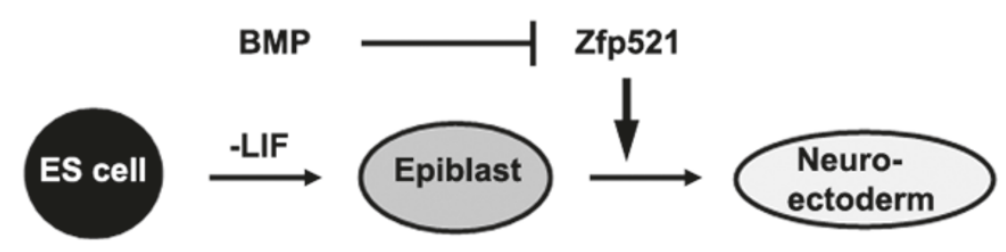 \\ (b)

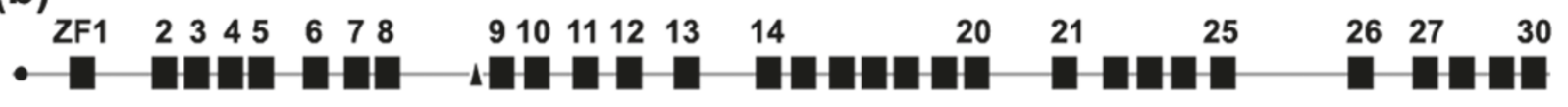 \\ (c) \\ p300 binding region \\ HDAC binding motif \\ GATA-1 binding region \\ EBF1 binding region? \\ Figure 1. Zfp521 structure and function. (a) In mouse embryonic stem (ES) cells, Zfp521 is essential for directing epiblasts to neuroectoderm cells, which is inhibited by bone morphogenetic protein (BMP) signaling pathways. (b) Schematic presentation of the Zfp521 protein, which contains a nuclear remodeling and histone deacetylation complex (NuRD) motif $(\bullet)$ at the N-terminal end, $30 \mathrm{C}_{2} \mathrm{H}_{2}$ zinc fingers (ZF1 to ZF30, $\mathbf{\square}$ throughout the protein, and a putative nuclear localization signal ( $\mathbf{\Delta}$ ) between ZF8 and ZF9. (c) Zfp521 recruits histone deacetylase (HDAC1/ HDAC3/HDAC4) through the NuRD motif, associates with co-activator p300 via ZF1 to ZF8, and inhibits GATA-1 and EBF1 by the C-terminal regions, and binds RunX2 through ZF6 to ZF26. LIF, leukemia inhibitory factor.}

Zfp521 by shRNA had no effect on regulation of ES cell markers Rex 1 and Nanog, but halted cells at the epiblast stage with sustained epiblast marker gene expression including Oct4, Cripto and Fgf5. Meanwhile Zfp521 depletion suppressed early neural gene induction and neural differentiation. Zfp521 is therefore critical for epiblast-neuroectoderm transition (Figure 1a) [7].

\section{Zfp521 structural domains and binding partners}

Zfp521 is a nuclear protein with a putative nuclear localization signal (seven amino acids) immediately upstream of ZF9. The protein contains 30 Krüppel-like ZF motifs clustered in five to seven ZFs throughout the protein (Figure 1b), interacts with multiple partners (Figure 1c), and regulates transcription in diverse tissues and organs. In ES cells, Zfp521 associates with p300 through ZF1 to ZF8 at the N-terminus to activate early neural genes and trigger neural differentiation [7]. In the hematopoietic system, its human counterpart ZNF521 binds and inhibits GATA-1 via ZF21 to ZF26, and this maintains the stemness of hematopoietic progenitors and represses erythroid differentiation [8]. Depletion of ZNF521 by shRNA can transform K562 and HEL cells into erythroid cells synthesizing hemoglobin and glycophorin A. The Cterminus of ZNF521 is also required for interaction/ inhibition of OLF1/EBF1, and the latter is important for activating B-cell-specific gene expression [9] and for the development of striatal medium spiny neurons [10]. Zfp521 also associates and attenuates RunX2 via ZF6 to ZF26 [11,12], and regulates two stages of osteoblast development during mesenchymal cell lineage commitment and during osteoblast maturation $[12,13]$.

Additionally, Zfp521 is capable of implementing epigenetic effects through global remodeling of the nucleus. This is achieved via a 12 -amino-acid motif called nuclear remodeling and histone deacetylation complex (NuRD) at its $\mathrm{N}$-terminal end, which binds histone deacetylases (HDACs) [14]. The general view is that HDACs deacetylate histones and repress gene transcription. For example, ZNF521 inhibits GATA-1 through NuRD, and deletion of the motif results in reduction of the inhibitory effect in hematopoietic progenitors [8]. The NuRD motif is highly conserved among other ZF transcription factors, including ZNF423, Friend of GATA (FOG-1/ZFPM1 and FOG-2/ZFPM2), BCL11A, and SALL family members [14], and may recruit different HDACs in different cells. In chondrocytes, Zfp521 forms a complex with HDAC3/ HDAC4 and inhibits RunX2 activity $[11,12]$. In ES cells Zfp521 antagonizes HDAC1/HDAC3, which inhibit p300-induced neural differentiation. Whether Zfp521, HDACs and p300 form one complex, however, is unclear. One scenario can be that association of p300 and Zfp521 results in either inactivation or release of HDACs from the complex. 
Interestingly, an N-terminally truncated ZNF521 isoform is predicted in human (uc002kvl.2) and rat (NP_001100873), which lacks the first 220 amino acids including the NuRD motif and ZF1 to ZF5. Meanwhile, the $\mathrm{NuRD}$ domain is neither required for the activation of $N$ cadherin expression in ES cells [7] nor for the inhibition of EBF1 in the hematopoietic system [9]. Information remaining to be validated includes whether the short isoform exists or is conserved during evolution, whether Zfp521 isoforms are expressed in different tissues, and whether the NuRD-truncated form is constitutively active as a result of its incapability to bind HDACs or is dominant negative as a result of missing ZF1 to ZF5.

\section{Zfp521 expression and regulation}

As a key intrinsic factor driving neural determination, we expect that Xenopus xZfp521 would be expressed at the early gastrula stage, such as st10.5, when neural induction is initiated by the Spemann organizer. Surprisingly, $x Z f p 521$ mRNA was not detected at st10.5 by in situ hybridization [7]. In mouse embryos, Zfp521 transcripts were not detected in blastocysts as anticipated, but were expressed weakly in E6.5 and strongly in E7.0 neuroectoderm during gastrulation. Whether Zfp521 is expressed in early postimplantation mouse embryos (E3.5 to E6.5) remains to be seen. Forced Zfp521 expression could not drive neural differentiation in the presence of leukemia inhibitory factor or the fibroblast growth factor receptor inhibitor SU5402, suggesting that Zfp521 may be a key factor for sustaining rather than initiating neural differentiation.

Understanding how Zfp521 is regulated is equally important. In ES cells it is currently unknown whether Zfp521 expression is purely repressed by BMP signaling, or can be induced by any other factors. What is known is that Zfp521 expression in chondrocytes can be increased by parathyroid hormone-related peptide (PTHrP) signaling or elevated adenylyl cyclase activity [11]. Knock-in of a GFP reporter into the $Z f p 521$ locus of ES cells with a similar approach may indicate whether any of the 104 genes highly expressed in Sox1-GFP cells is upstream of the $Z f p 521$. Alternatively, a quantitative RT-PCR analysis of the genes in a time course of the 3-day neural induction could indicate whether any factor was induced prior to $Z f p 521$. It will also be interesting to know whether WNT signaling pathways [15], downstream of the adenylyl cyclase pathway such as protein kinase A, or a well-known neural inducer such as retinoic acid may influence Zfp521 expression.

\section{Zfp521 function}

Zfp521 is expressed in a wide range of tissues including the brain, muscle, heart, kidney, spleen, lymph nodes, placenta, thymus and fetal liver [16]; in particular, in immature cells such as early mesenchymal condensations, developing bones, hematopoietic stem cells, and neural progenitors. Zfp521 was initially called Evi3 in mouse, which was identified as a common integration site of retrovirus in murine AKXD B-cell lymphomas [17]. The virus integrates upstream of the first coding exon, which results in upregulation of Evi3. The human ZNF521 was previously termed early hematopoietic zinc finger protein, and its expression in the hematopoietic system was restricted to a subpopulation of less differentiated CD34+ cells. ZNF521 is abundant in leukemia, medulloblastomas and other brain tumors. iRNA-mediated ZNF521 silencing impairs the growth and clonogeneity of $\mathrm{MLL}^{+} / \mathrm{ZNF} 521^{+}$THP-1 cells. High levels of Zfp521 expression may therefore be associated with oncogenesis.

Zfp521 is shown to regulate a number of cellular processes. The protein promotes proliferation, delays differentiation and reduces apoptosis [13]. In cultured mesenchymal cells, Zfp521 expression is decreased by BMP2 and increased by PTHrP, which promote and antagonize osteoblast differentiation, respectively. Deleting $Z f p 521$ also decreases cyclin $\mathrm{D}_{1}$ and Bcl-2 expression with increased caspase-3 activation and apoptosis. Interestingly, in stage 15 of Xenopus embryos developed from injection of $x Z f p 521$ expression vector at the eight-cell stage, an altered distribution pattern of cells expressing pro-neural markers Neurogenin and Estrogen Receptor 1 is observed [7]. This observation suggests that Zfp521 overexpression may induce ectopic expression of pro-neural genes. Alternatively, appropriate levels of Zfp521 expression may be essential for neuronal migration. Zfp521 expression in adult brain is very limited, however, based on the Allen Brain Atlas [18] in comparison with developing neural tubes [7], whereas relatively high levels of the mRNAs for Zfp521 and its partner Ebf1 are detected in striatonigral neurons of the adult mouse [10] - Zfp521 may therefore be involved in neurodegenerative and psychiatric disorders.

In summary, Zfp521 plays important roles in multiple tissues. Zfp521 mediates PTHrP signaling, and regulates proliferation and differentiation of growth plate chondrocyte [11] and bone formation [13]. In embryos, Zfp521 is critical for rostral neural tube development, because Zfp521-depleted ES cells fail to contribute to the rostral neural tube, although they are found in other parts of chimeric embryos [7]. Investigation of $Z f p 521^{-/-}$mice will reveal how Zfp521 affects brain development. The excellent work carried out by Kamiya and colleagues demonstrates that Zfp521 is a key factor to sustain neural differentiation. These findings have opened up a whole series of studies to investigate how Zfp521 works together with other transcriptional factors and epigenetic factors to regulate neural differentiation in stem cells and in developing brains of mice and men. 


\section{Abbreviations}

BMP, bone morphogenetic protein; ES, embryonic stem; FCS, fetal calf serum GFP, green fluorescence protein; HDAC, histone deacetylase; iRNA, interfering RNA; NuRD, nuclear remodeling and histone deacetylation complex; PCR, polymerase chain reaction; PTHrP, parathyroid hormone-related peptide; RT, reverse transcription; shRNA, short hairpin RNA; ZF, zinc finger.

\section{Competing interests}

The authors declare that they have no competing interests.

\section{Published: 27 April 2011}

\section{References}

1. Thomson JA, Itskovitz-Eldor J, Shapiro SS, Waknitz MA, Swiergiel JJ, Marshall VS, Jones JM: Embryonic stem cell lines derived from human blastocysts. Science 1998, 282:1145-1147.

2. Takahashi K, Yamanaka S: Induction of pluripotent stem cells from mouse embryonic and adult fibroblast cultures by defined factors. Cell 2006 126:663-676.

3. Yuan X, Wan H, Zhao X, Zhu S, Zhou Q, Ding S: Combined chemical treatment enables Oct4-induced reprogramming from mouse embryonic fibroblasts. Stem Cells 2011, 29:549-553.

4. Spemann $\mathrm{H}$, Mangold $\mathrm{H}$ : Über induktion von embryonanlagen durch implantation artfremder organisatoren. Wilhelm Roux Arch Entw Mech Organ 1924, 100:599-638.

5. Muñoz-Sanjuán I, Brivanlou AH: Neural induction, the default model and embryonic stem cells. Nat Rev Neurosci 2002, 3:271-280.

6. Walsh DW, Godson C, Brazil DP, Martin F: Extracellular BMP-antagonist regulation in development and disease: tied up in knots. Trends Cell Biol 2010, 20:244-256.

7. Kamiya D, Banno S, Sasai N, Ohgushi M, Inomata H, Watanabe K, Kawada M, Yakura R, Kiyonari H, Nakao K, Jakt LM, Nishikawa S, Sasai Y: Intrinsic transition of embryonic stem-cell differentiation into neural progenitors. Nature 2011, 470:503-509.

8. Matsubara E, Sakai I, Yamanouchi J, Fujiwara H, Yakushijin Y, Hato T, Shigemoto K, Yasukawa M: The role of zinc finger protein 521/early hematopoietic zinc finger protein in erythroid cell differentiation. J Biol Chem 2009, 284:3480-3487.

9. Bond HM, Mesuraca M, Amodio N, Mega T, Agosti V, Fanello D, Pelaggi D,
Bullinger L, Grieco M, Moore MA, Venuta S, Morrone G: Early hematopoietic zinc finger protein-zinc finger protein 521: a candidate regulator of diverse immature cells. Int J Biochem Cell Biol 2008, 40:848-854.

10. Lobo MK, Yeh C, Yang XW: Pivotal role of early B-cell factor 1 in development of striatonigral medium spiny neurons in the matrix compartment. J Neurosci Res 2008, 86:2134-2146.

11. Correa D, Hesse E, Seriwatanachai D, Kiviranta R, Saito H, Yamana K, Neff L, Atfi A, Coillard L, Sitara D, Maeda Y, Warming S, Jenkins NA, Copeland NG, Horne WC, Lanske B, Baron R: Zfp521 is a target gene and key effector of parathyroid hormone-related peptide signaling in growth plate chondrocytes. Dev Cell 2010, 19:533-546.

12. Hesse E, Saito H, Kiviranta R, Correa D, Yamana K, Neff L, Toben D, Duda G, Atf A, Geoffroy V, Horne WC, Baron R: Zfp521 controls bone mass by HDAC3dependent attenuation of Runx2 activity. J Cell Biol 2010, 191:1271-1283.

13. Wu M, Hesse E, Morvan F, Zhang JP, Correa D, Rowe GC, Kiviranta R, Neff L, Philbrick WM, Horne WC, Baron R: Zfp521 antagonizes Runx2, delays osteoblast differentiation in vitro, and promotes bone formation in vivo. Bone 2009, 44:528-536.

14. Lin AC, Roche AE, Wilk J, Svensson EC: The N termini of Friend of GATA (FOG) proteins define a novel transcriptional repression motif and a superfamily of transcriptional repressors. J Biol Chem 2004, 279:55017-55023.

15. Liu J, Bang AG, Kintner C, Orth AP, Chanda SK, Ding S, Schultz PG: Identification of the Wnt signaling activator leucine-rich repeat in Flightless interaction protein 2 by a genome-wide functional analysis. Proc Natl Acad Sci U S A 2005, 102:1927-1932.

16. Warming S, Liu P, Suzuki T, Akagi K, Lindtner S, Pavlakis GN, Jenkins NA, Copeland NG: Evi3, a common retroviral integration site in murine B-cell lymphoma, encodes an EBFAZ-related Kruppel-like zinc finger protein. Blood 2003, 101:1934-1940.

17. Justice MJ, Morse HC 3rd, Jenkins NA, Copeland NG: Identification of Evi-3, a novel common site of retroviral integration in mouse AKXD B-cell lymphomas. J Virol 1994, 68:1293-1300.

18. Allen Brain Atlas [http://www.brainatlas.org/]

\section{doi:10.1186/scrt61}

Cite this article as: Shen $\mathrm{S}$, et al:: A zinc finger protein Zfp521 directs neural differentiation and beyond. Stem Cell Research \& Therapy 2011, 2:20. 